\title{
Efficacy and safety of bevacizumab-containing therapy for refractory advanced breast cancer: a retrospective study
}

\author{
Huimin Lv^, Limin Niu, Mengwei Zhang, Huiai Zeng, Shengnan Zhao, Jing Wang, Huihui Sun, \\ Zhenzhen Liu, Min Yan
}

Department of Breast Disease, Henan Breast Cancer Center, Affiliated Cancer Hospital of Zhengzhou University \& Henan Cancer Hospital, Zhengzhou, China

Contributions: (I) Conception and design: M Yan; (II) Administrative support: Z Liu, M Yan; (III) Provision of study materials or patients: All authors; (IV) Collection and assembly of data: H Lv, L Niu, M Zhang, S Zhao, J Wang, H Sun; (V) Data analysis and interpretation: H Lv, H Sun, M Yan; (VI) Manuscript writing: All authors; (VII) Final approval of manuscript: All authors.

Correspondence to: Min Yan. Department of Breast Disease, Henan Breast Cancer Center, Affiliated Cancer Hospital of Zhengzhou University \& Henan Cancer Hospital, Zhengzhou, China. Email: ym200678@126.com.

\begin{abstract}
Background: Advanced breast cancer (ABC) is difficult to treat due to the primary and acquired resistance, which is the main cause of rescue treatment failure. Thus, developing a new rescue treatment strategy for clinicians is a challenge. This study retrospectively collected the medical information of patients with refractory $\mathrm{ABC}$ who were treated with bevacizumab at our department to analyze the efficacy and safety of bevacizumab-containing therapy as a new treatment method for refractory ABC.

Methods: The complete medical information of patients receiving bevacizumab treatment from November 2017 to November 2020 was collected, and patients' general medical history and disease characteristics were analyzed. The objective response rate (ORR), disease control rate (DCR), progression-free survival (PFS), time to failure (TTF), overall survival (OS), and safety were also analyzed using SPSS 20.0 statistical software.

Results: The data of 68 women with refractory ABC who received bevacizumab-containing therapy were collected. The last follow-up examinations were performed on March 31, 2021. The ORR was 41.2\% and the DCR was $88.2 \%$. The median PFS, TTF, and OS were 6.0 months [95\% confidence interval (CI): 3.4-8.6 months], 4.0 months (95\% CI: 3.2-4.8 months), and 26.6 months (95\% CI: 10.2-43.0 months), respectively. Treatment was discontinued in 2 patients due to chemo-related adverse reactions. The main adverse events related to bevacizumab were hypertension (7.4\%) and bleeding (2 cases, $2.9 \%$ ), both of which were grade 1 . No specific adverse events causing the discontinuation of bevacizumab treatment were observed in the other patients.

Conclusions: Among patients with refractory ABC, bevacizumab-containing therapy is feasible and safe, and can be used as a new treatment strategy. However, the question of who can benefit the most from bevacizumab-containing therapy requires further exploration.
\end{abstract}

Keywords: Bevacizumab; antiangiogenic therapy; refractory; advanced breast cancer (ABC)

Submitted Aug 31, 2021. Accepted for publication Oct 20, 2021.

doi: $10.21037 /$ apm-21-2745

View this article at: https://dx.doi.org/10.21037/apm-21-2745

$\wedge$ ORCID: 0000-0002-4808-5782. 


\section{Introduction}

Currently, chemotherapy, radiotherapy, endocrine therapy, and anti-human epidermal growth factor receptor 2 (HER2) targeted therapy are commonly used to treat advanced breast cancer (ABC) (1). However, the emergence of multidrug resistance can cause systemic anti-tumor failure and prolonged treatment times. Thus, a more effective treatment method for $\mathrm{ABC}$ is needed.

Angiogenesis is the most critical step in the development and progression of malignant tumors. The same is true for breast cancer metastasis. The effective inhibition of tumor angiogenesis can inhibit the growth of tumor cells and reduce the occurrence of metastasis. Thus, antiangiogenic therapy represents a novel treatment for drug-resistant tumors (2). Apatinib is a new antiangiogenic drug whose efficacy in $\mathrm{ABC}$ has been confirmed by several studies (3-5). However, adverse reactions, such as hypertension and proteinuria, have limited its broad clinical application (3-5).

Bevacizumab is an anti-vascular endothelial growth factor (VEGF) monoclonal antibody. In the $1^{\text {st }}$ line of treatment, bevacizumab combined with chemotherapy significantly improves the objective response rate (ORR) and progression-free survival (PFS) of patients with advanced triple-negative breast cancer $(6,7)$. This combination therapy has also been shown to be well tolerated with few adverse reactions $(6,7)$. Thus, the question of whether bevacizumab combined with chemotherapy is an effective treatment strategy for patients with multi-drug resistant, refractory $\mathrm{ABC}$ requires further investigation. Besides, this study provides data about bevacizumab in combination with non-taxane containing regimens, such as, Vinorelbine, Gemcitabine, Cisplatin, Capecitabine, Eribulin or Epirubicin. We present the following article in accordance with the STROBE reporting checklist (available at https:// dx.doi.org/10.21037/apm-21-2745).

\section{Methods}

\section{Data}

We analyzed the medical history of patients with refractory $\mathrm{ABC}$ who received bevacizumab (Avastin ${ }^{\circledR}$ ) combined with chemotherapy at the Department of Mammary Surgery, Henan Cancer Hospital from November 2017 to November 2020. All procedures performed in this study involving human participants were in accordance with the Declaration of Helsinki (as revised in 2013). The study was approved by the medical ethics committee of Henan Cancer
Hospital (No. 2107407) and informed consent was taken from all the patients.

In this retrospective study, refractory $\mathrm{ABC}$ was defined as follows: disease progression during neoadjuvant chemotherapy or adjuvant chemotherapy, or relapse and rapid progression within 1 year of adjuvant chemotherapy, or disease progression after multi-line standard rescue therapy for which no standard drugs are available.

To be eligible to participate in this study, patients had to meet the following inclusion criteria:

(I) Have pathologically confirmed invasive breast cancer;

(II) Have local recurrence or metastasis that cannot be resected;

(III) Have estrogen receptors (ER), progesterone receptors (PR), and HER2 in the primary tumor and/or metastatic lesions;

(IV) Have experienced failure or the rapid progression of the disease after standard rescue treatment;

(V) Have a physical status of 0-2 based on the Eastern Cooperative Oncology Group (ECOG) scoring system;

(VI) Have undergone bevacizumab treatment for at least 1 cycle, and have follow-up data available regarding the efficacy and safety of this treatment; and

(VII) Have an expected life period of $\geq 12$ weeks.

Patients were excluded from the study if they met any of the following exclusion criteria:

(I) The presence of ER, PR, and HER2 in the primary breast lesions or metastatic lesions was unknown; and/or

(II) Follow-up data about the safety and efficiency of the bevacizumab treatment were unavailable.

A total of 68 female patients were included in the study. The patients had a median age of 50 years (range, 28-70 years). Thirty-seven patients were premenopausal, and 31 were postmenopausal. There were 52 cases of visceral metastasis, and 16 cases of non-visceral metastasis. There were 33 cases of liver metastasis and 35 cases of non-liver metastasis. The median treatment line was 2.5 (range, 2-16); there were 17 cases of 2nd-line treatment, 17 cases of 3 rd-line treatment, and 34 cases of 4th-line or above treatment; 31 and 37 patients received combined chemotherapy and single-drug chemotherapy, respectively. Chemotherapeutic agents that have not been shown to be resistant during prior treatment, such as gemcitabine, Vinorelbine, gemcitabine, docetaxel, eribulin, cisplatin, albumin bound paclitaxel, capecitabine, epirubicin are all 
Table 1 Clinical characteristics of 68 patients with advanced breast cancer

\begin{tabular}{|c|c|}
\hline Factor & $\mathrm{N}(\%)$ \\
\hline Age (years) & 50 [28-70] \\
\hline \multicolumn{2}{|l|}{ ECOG } \\
\hline 0 & $26(38.2)$ \\
\hline 1 & $37(54.4)$ \\
\hline 2 & $5(7.3)$ \\
\hline \multicolumn{2}{|l|}{ Menstrual status } \\
\hline Postmenopausal & $34(50.0)$ \\
\hline Premenopausal & $34(50.0)$ \\
\hline \multicolumn{2}{|l|}{ Age (years) } \\
\hline$\leq 40$ & $18(26.5)$ \\
\hline$>40$ & $50(73.5)$ \\
\hline \multicolumn{2}{|l|}{ Chemotherapy } \\
\hline Combined chemotherapy & $31(45.5)$ \\
\hline Single-drug chemotherapy & $37(54.5)$ \\
\hline \multicolumn{2}{|l|}{ Classification } \\
\hline Triple-negative & $30(44.1)$ \\
\hline Luminal (HER2-) & $28(41.2)$ \\
\hline Luminal (HER2+) & $8(11.8)$ \\
\hline HER2 amplification & $2(2.9)$ \\
\hline \multicolumn{2}{|l|}{ DFI (years) } \\
\hline$\geq 1$ & $31(45.6)$ \\
\hline$<1$ & $26(38.2)$ \\
\hline De novo metastatic & $11(16.2)$ \\
\hline \multicolumn{2}{|l|}{ Visceral metastasis } \\
\hline Yes & $52(76.4)$ \\
\hline No & $16(23.6)$ \\
\hline \multicolumn{2}{|l|}{ HER2 status } \\
\hline Positive & $10(14.7)$ \\
\hline Negative & $58(85.3)$ \\
\hline \multicolumn{2}{|l|}{ Metastasis site } \\
\hline Liver & $33(48.5)$ \\
\hline Lung & $30(44.1)$ \\
\hline Lymph node & $37(54.4)$ \\
\hline
\end{tabular}

Table 1 (continued)
Table 1 (continued)

\begin{tabular}{lc}
\hline Factor & $\mathrm{N}(\%)$ \\
\hline Bone & $27(39.7)$ \\
Chest wall & $22(32.4)$ \\
Bone marrow & $2(2.9)$ \\
Pleura & $13(19.1)$ \\
Soft tissue & $2(2.9)$ \\
Pericardium & $1(1.5)$ \\
Breast & $4(5.9)$ \\
Brain & $7(10.3)$ \\
\hline DFl, disease-free interval.
\end{tabular}

acceptable. There were 30 cases of triple-negative breast cancer, 28 cases of HR+/HER2-, 8 cases of HR+/HER2+, and 2 cases of HR-/HER2+ (see Table 1). The treatment line was defined as the number of times the patients received rescue treatment after metastatic breast cancer was diagnosed or after the first diagnosis of stage IV breast cancer. Patients who showed disease progression during (neo)adjuvant chemotherapy or recurrence within 1 year after completing adjuvant chemotherapy were considered to have received 1st-line treatment.

\section{Study details}

\section{Treatment}

All the patients were treated with bevacizumab-based combination therapy. Bevacizumab (Avastin ${ }^{\circledR}, 4 \mathrm{~mL} / 100 \mathrm{mg}$ ) was administered at $5 \mathrm{mg} / \mathrm{kg}$ per dose, once per week in each 21-day cycle. Chemotherapeutic drugs were administered according to the dosage recommended in the Chinese Society of Clinical Oncology (CSCO): Clinical guidelines for the diagnosis and treatment of breast cancer published in 2020. Details about the administration of the chemotherapy cycles are summarized in Table 2.

Patients' blood pressure and liver and kidney function were monitored during treatment. Routine blood tests were also performed. The principles of dose reduction and drug withdrawal were as follows: when grade 4 adverse events occurred for the 1st time or grade 3 hematological toxicity occurred for twice consecutively, the corresponding chemotherapy or bevacizumab was reduced to 1 dose 
Table 2 The drug exposure cycle number of 68 patients with advanced breast cancer

\begin{tabular}{|c|c|c|}
\hline Combination regimen & N (\%) & The number of cycles \\
\hline Vinorelbine + Cisplatin & $17(25.0)$ & $6[3-8]$ \\
\hline Gemcitabine + Cisplatin & $8(11.8)$ & $6[1-8]$ \\
\hline Docetaxel + Cisplatin & $4(5.9)$ & $4.5[2-6]$ \\
\hline Eribulin + Cisplatin & $1(1.5)$ & 1 \\
\hline \multicolumn{3}{|l|}{ Single-drug chemotherapy } \\
\hline Gemcitabine & $14(20.6)$ & $5[1-11]$ \\
\hline Vinorelbine & $6(8.8)$ & $3[1-10]$ \\
\hline Docetaxel & $3(4.4)$ & $4[3-6]$ \\
\hline Eribulin & $3(4.4)$ & $4[4-6]$ \\
\hline Epirubicin & $1(1.5)$ & 5 \\
\hline Bevacizumab & $68(100.0)$ & $5[1-26]$ \\
\hline Combined with trastuzumab & $4[40]$ & $5[3-10]$ \\
\hline
\end{tabular}

level after recovery to $\leq$ grade 1 ; if the adverse event was intolerable, the patients were asked to stop the medication. The treatment endpoints were as follows: disease progression, or intolerable toxicity, or termination of treatment for any reason, or death from any cause.

\section{Measurement of outcomes}

The response evaluation criteria in solid tumors (RECIST, version 1.1), including complete response (CR), partial response (PR), stable disease (SD) and progressive disease (PD) (8) was used to evaluate treatment efficacy every 2-3 treatment cycles. The primary study endpoints included ORR, disease control rate (DCR), PFS, time to failure (TTF), overall survival (OS), and safety, where PFS was defined as the time from the beginning of bevacizumab treatment to disease progression or death from any cause, TTF was defined as the time from the beginning of bevacizumab treatment to treatment interruption or loss of follow-up due to any reason, OS was defined as the time from the beginning of bevacizumab treatment to death from any cause. The classification of adverse time was based on Common Terminology Criteria for Adverse (CTC-AE) 4.03 by the National Cancer Institute.

\section{Statistical analysis}

SPSS 20.0 software was used for the statistical analysis. The Kaplan-Meier method was used for the survival analysis. The log-rank test was used for the univariate analysis of the prognostic factors between the two groups. A Cox proportional-hazards regression model was constructed for the multivariate analysis. A $\mathrm{P}$ value of $<0.05$ was considered statistically significant (bilateral).

\section{Results}

\section{Efficacy}

Sixty-eight patients participated in the study. Of these, PD was observed in 35 patients. The last follow-up time was March 31, 2021, and the median follow-up time was 26.0 months. Four patients were lost during the follow-up period, and 3 patients continued to receive bevacizumab treatment. All patients had at least 1 measurable lesion. Specifically, there was 1 case of CR, 27 cases of PR, 32 cases of SD, and 8 cases of PD. The ORR was $41.2 \%$, and the DCR was $88.2 \%$. The median PFS, TTF, and OS were 6.0 months [95\% confidence interval (CI): 3.4-8.6 months], 
4.0 months (95\% CI: 3.2-4.8 months), and 26.6 months (95\% CI: 10.2-43.0 months), respectively (see Table 3 and Figure 1).

The ORR univariate analysis showed that combined chemotherapy was better than single-drug chemotherapy $(\mathrm{P}=0.01)$, but no difference was observed in different age, metastasis site, the number of metastatic sites, or treatment line $(\mathrm{P}>0.05$; see Table 4$)$. The PFS univariate analysis showed that the treatment efficacy of chemotherapy combined with bevacizumab for patients with refractory $\mathrm{ABC}$ was not significantly correlated with menstrual status, metastasis site, disease-free interval (DFI), the number of metastatic sites, treatment line, combined chemotherapy mode, and PFS $(\mathrm{P}>0.05)$. The OS univariate analysis showed

Table 3 The efficacy observation of 43 evaluable cases

\begin{tabular}{|c|c|}
\hline Factor & N (\%) \\
\hline $\mathrm{CR}$ & $1(1.5)$ \\
\hline PR & $27(39.7)$ \\
\hline SD & $32(47.1)$ \\
\hline PD & $8(11.8)$ \\
\hline ORR & $28(41.2)$ \\
\hline DCR & $60(88.2)$ \\
\hline Median PFS (months) & 6.0 (95\% Cl: 3.4-8.6) \\
\hline Median TTF (months) & 4.0 (95\% Cl: 3.2-4.8) \\
\hline Median OS (months) & 26.6 (95\% Cl: 10.2-43.0) \\
\hline $\begin{array}{l}\text { CR, complete respo } \\
\text { disease; PD, progressi } \\
\text { DCR, disease control } \\
\text { time to failure; OS, ove }\end{array}$ & $\begin{array}{l}\text { partial response; SD, stable } \\
\text {; ORR, objective response rate; } \\
\text { progression-free survival; TTF, } \\
\text { al. }\end{array}$ \\
\hline
\end{tabular}

A

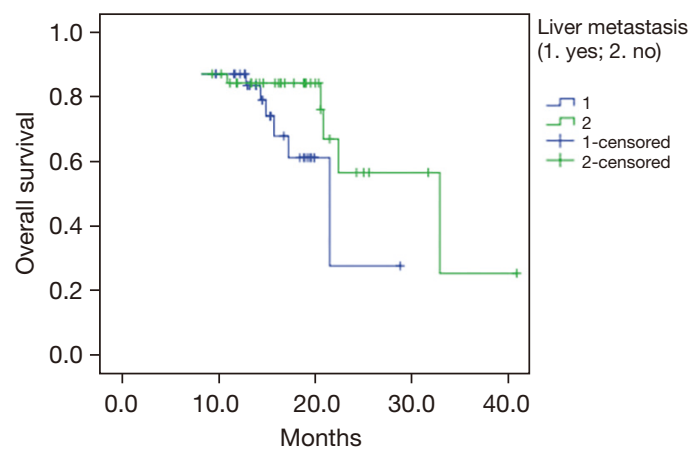

that the $\mathrm{OS}$ of patients with refractory $\mathrm{ABC}$ who received chemotherapy combined with bevacizumab was associated with age and liver metastasis $(\mathrm{P}<0.05)$. The results showed that the OS was 13.3 and 26.6 months in the $\leq 40$-year-old and $>40$-year-old groups $(\mathrm{P}=0.030)$, respectively, whereas the OS was 14.3 and 26.6 months in patients with and without liver metastasis, respectively ( $\mathrm{P}=0.047$; see Figure 1). There was no significant correlation between the OS and the number of metastatic sites, treatment lines, molecular classification, and combined treatment mode $(\mathrm{P}>0.05)$. However, the multivariate analysis showed that the efficacy of bevacizumab combined with chemotherapy was not associated with age or liver metastasis $(\mathrm{P}>0.05$; see Table 5).

There were 65 TTF events, including disease progression $(50.8 \%)$, financial burden (38.5\%), intolerable adverse reactions $(3.1 \%)$, loss to follow-up (6.2\%), and discontinuation of the drug after medical approval because surgery was required $(1.5 \%)$. Thus, other than disease progression, the financial burden caused by the disease was the leading cause of treatment discontinuation.

\section{Safety}

Adverse events in at least 1 treatment cycle were observed in 68 patients. During the treatment, 2 patients discontinued because of adverse reactions, including thrombocytopenia and increased alanine aminotransferase level, which might be associated with chemotherapy. We obtained information about bevacizumab-related adverse events from all patients. There were 5 cases $(7.4 \%)$ of hypertension, including 4 cases $(5.9 \%)$ of grade 2 hypertension, and 1 case $(1.5 \%)$ of grade 3 hypertension. There were 2 cases $(2.9 \%)$ of hemorrhage, both of which

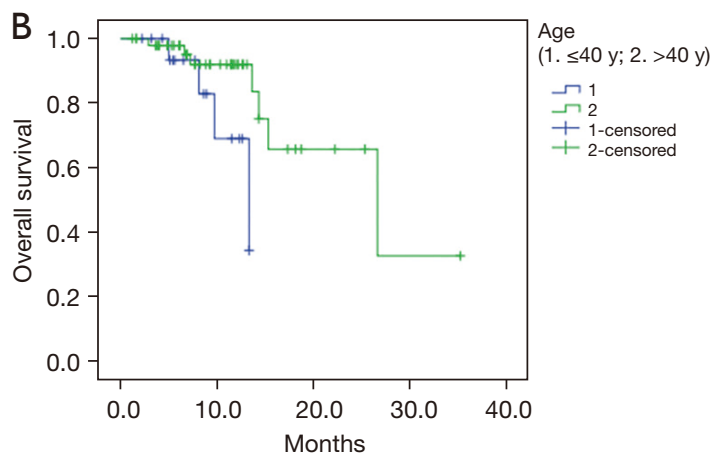

Figure 1 Survival curve of univariate analysis of OS after treatment with bevacizumab-containing therapy [(A) $\mathrm{P}=0.047$, (B) $\mathrm{P}=0.030]$. OS, overall survival. 
Table 4 The ORR and univariate analysis of 68 patients with advanced breast cancer

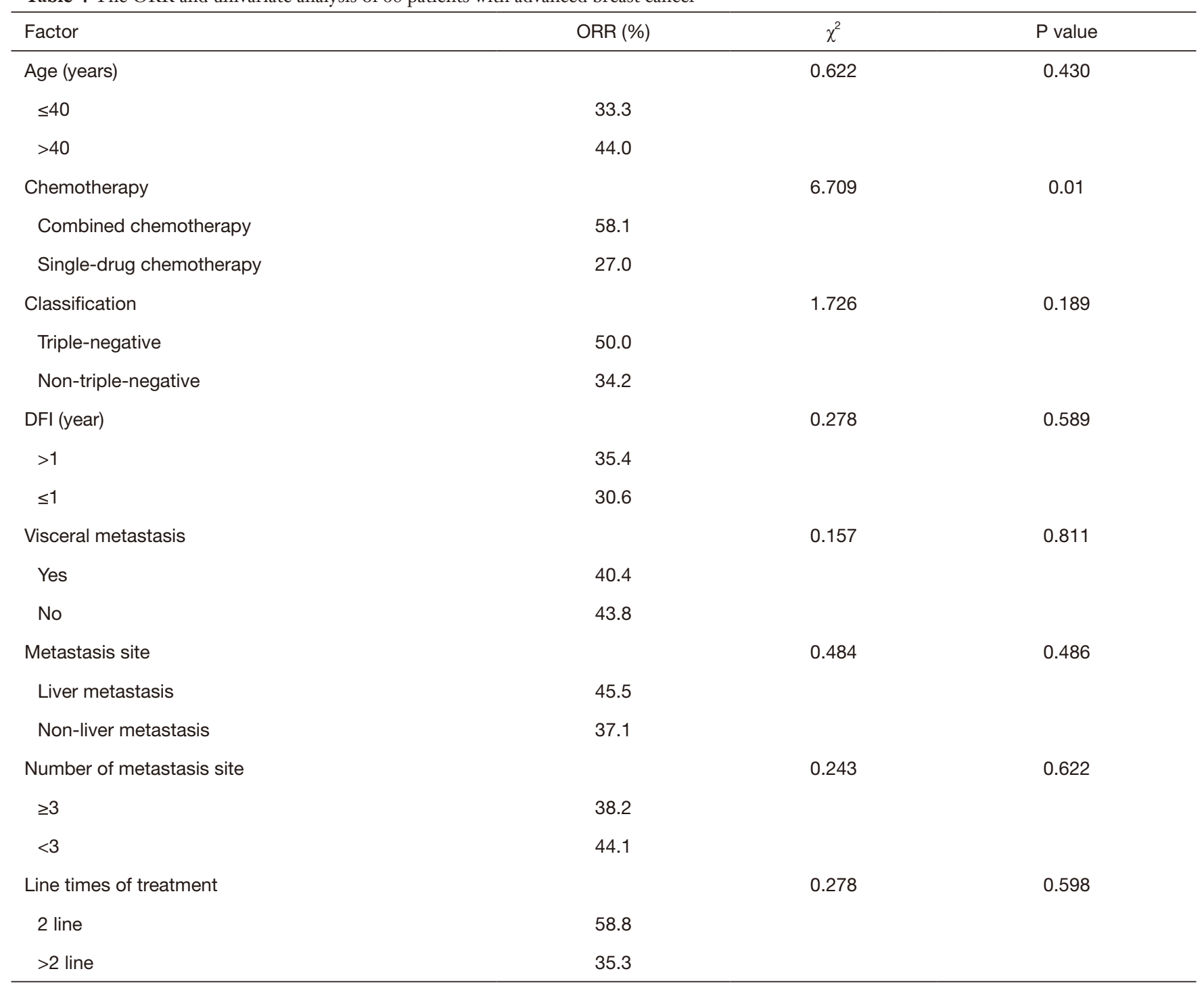

ORR, objective response rate.

Table 5 Multivariate analysis of OS of bevacizumab treatment combined with chemotherapy

\begin{tabular}{lccc}
\hline Factor & $\mathrm{B}$ & $\mathrm{P}$ value & $95 \% \mathrm{Cl}$ \\
\hline Chemotherapy (combined or single drug) & -1.067 & 0.137 & $0.344(0.084-1.406)$ \\
Liver metastasis (1. yes; 2. no) & 0.804 & 0.389 & $2.234(0.358-13.928)$ \\
Age (1. $\leq 40$ years; $2 .>40$ years) & 1.556 & 0.050 & $4.739(0.997-22.529)$ \\
Metastasis site (1. $\geq 3$ sites; $2 .<2$ sites) & 0.294 & 0.726 & $1.342(0.259-6.958)$ \\
\hline
\end{tabular}

OS, overall survival. 
were grade 1 and improved after symptomatic treatment. No patient discontinued the treatment because of bevacizumab-related adverse events.

\section{Discussion}

In this study, we retrospectively analyzed the efficacy and safety of bevacizumab in patients with refractory ABC. The ORR and DCR were $41.2 \%$ and $88.2 \%$, respectively in 68 patients who received bevacizumab combined with chemotherapy. The median PFS and OS were 6.0 and 26.6 months, respectively, without severe adverse reactions. Thus, our results showed that bevacizumab combined with chemotherapy is feasible for treating refractory $\mathrm{ABC}$.

About $90 \%$ of primary tumors and $50 \%$ of metastatic tumors are sensitive in the early stage of treatment; however, with the extension of treatment time, tumors tend to become resistant to drugs (9). Studies have sought to understand the mechanism underlying drug resistance. The phosphatidylinositol 3-kinase/protein kinase B/ mammalian target of rapamycin (PI3K/Akt/mTOR) pathway interacts with the ER signaling pathway. The activated $\mathrm{PI} 3 \mathrm{~K} / \mathrm{Akt} / \mathrm{mTOR}$ pathway promotes messenger ribonucleic acid (mRNA) transcription in the ER-mediated downstream signaling pathway and the proliferation of tumor cells (10). Similar to findings on ER positivity, the drug resistance mechanism of HER2-positive breast cancer is related to the abnormal activation of the PI3K/Akt/ mTOR signaling pathway. The continuous activation of the $\mathrm{PI} 3 \mathrm{~K} / \mathrm{Akt} / \mathrm{mTOR}$ signaling pathway can lead to the loss of phosphatase and tensin homolog function, thus activating the PI3K signaling pathway and reducing the efficiency of trastuzumab $(11,12)$. Previous studies have confirmed that everolimus, an mTOR inhibitor, reverses the acquired resistance of HR-positive $\mathrm{ABC}$ to tamoxifen or aromatase inhibitors (13-15). Similarly, for patients with HER2positive $A B C$, everolimus combined with trastuzumab and chemotherapy was found to improve PFS (16). In a study of triple-negative breast cancer resistance, 1st-line chemotherapy combined with bevacizumab, an angiogenesis inhibitor, was found to improve the PFS of patients (17). Some studies have confirmed that antiangiogenic therapy combined with immune checkpoint inhibitor treatment has a synergistic anti-tumor effect (18), and its clinical efficacy is being verified. In conclusion, the resistance mechanism of breast cancer is complex and clinical accessibility is poor. New anti-tumor drugs are required to further improve patient outcomes.
In 1971, Judah Folkman postulated the angiogenesis theory (19) that tumor growth and metastasis depended on tumor angiogenesis, and that inhibiting tumor angiogenesis played a major role in tumor treatment (20). VEGF and its vascular endothelial growth factor receptor (VEGFR)mediated signal transduction pathway play an important role in regulating tumor angiogenesis (21). Bevacizumab is the 1st approved VEGF inhibitor worldwide, and was approved in China in February 2010. Bevacizumab is a recombinant humanized monoclonal immunoglobulin $\mathrm{G} 1$ antibody and can specifically block the biological effect of VEGF, which inhibits the mitosis of vascular endothelial cells and reduces angiogenesis. Bevacizumab can bind to all subtypes of VEGF-A and prevent VEGF from binding to its receptor, thereby inhibiting angiogenesis and exudation (22). Clinical results have suggested that Bevacizumab combined with chemotherapy significantly improve the ORR and prolong the PFS time in patients with recurrent and metastatic breast cancer (23).

Apatinib, a novel, small molecule, VEGFR tyrosine kinase inhibitor, binds explicitly to VEGFR-2 in vivo to effectively control tumor angiogenesis and growth. Apatinib is efficacious in patients with multi-drug resistant $\mathrm{ABC}$. We previously studied the efficacy of apatinib monotherapy in patients with multi-drug resistant $\mathrm{ABC}$ (5), and found that the ORR $(43.5 \%)$ and DCR $(82.6 \%)$ were better than those reported in a previous study (3). It may be that a multi-drug resistant gene, a target gene of hypoxiainducible factor-1 (HIF-1 $\alpha$ ), can be induced by HIF- $1 \alpha$ under hypoxia conditions to enhance tumor resistance, and HIF- $1 \alpha$ is directly related to tumor angiogenesis. Antiangiogenic therapy may be an effective treatment strategy for multi-drug resistant breast cancer. However, for the long-term clinical application of apatinib, we must pay attention to the management of its adverse events. A high proportion of hypertension, proteinuria, and handfoot skin reaction limit its long-term clinical application (3-5). In the present study, we investigated the clinical application of bevacizumab combined with chemotherapy in patients with refractory ABC. The ORR and DCR were $41.2 \%$ and $88.2 \%$, respectively. The PFS and OS were 6.0 and 26.6 months, respectively. In this study, we focused on the adverse events associated with bevacizumab, with $7.4 \%$ of hypertension and $2.9 \%$ of hemorrhage, No patient discontinued the treatment because of bevacizumabrelated adverse events. Therefore, the short-term effective rate was similar to that of apatinib, but PFS and OS were better, with tolerable adverse reactions and manageable 
adverse events. The application of bevacizumab combined with chemotherapy is easy to promote in clinical practice. The univariate analysis revealed that the ORR of patients receiving bevacizumab combined with chemotherapy was better than that of single-drug chemotherapy without improved PFS and OS. Thus, combined chemotherapy can achieve a better disease remission rate, but its longterm benefits are limited. This finding is consistent with those reported previously (24). For patients with a large tumor load, obvious disease symptoms, and who need rapid treatment, a combination of chemotherapy with bevacizumab is recommended, which is consistent with the results of a previous study (24).

For patients with HER2-positive $\mathrm{ABC}$ who failed to receive multi-line anti-HER2 therapy, the benefit of readministering trastuzumab is limited. There were 10 cases of HER2-positive ABC. Among these 10 cases, there were 4 cases (comprising 2 cases of PR and 2 cases of SD) received combined treatment with trastuzumab, and 6 cases (comprising 3 cases of $\mathrm{PR}$ and 3 cases of $\mathrm{SD}$ ) received no anti-HER2 treatment. Thus, for patients with failure of anti-HER2 targeted therapy for primary drug resistance or failure of multi-line anti-HER2 treatment, the question of whether to continue anti-HER2 pathway treatment or to use anti-tumor drugs (e.g., antiangiogenic therapy) deserves clinical consideration and further investigation.

This study had some limitations. First, it was retrospective in nature and had a small sample size, and there was a selection bias, as the clinicians prescribed singledrug chemotherapy or combined chemotherapy. Second, in this study, the proportion of patients who discontinued treatment was high because of the financial burden, and the reduction in treatment duration could have reduced ORR and PFS. Third, the follow-up time was short, and further follow-up examinations are needed to determine the long-term survival of these patients and the safety of this combination therapy.

\section{Conclusions}

By performing a retrospective analysis of practical data, we confirmed the efficacy and safety of bevacizumab combined with chemotherapy in treating refractory $\mathrm{ABC}$, thus providing evidence supporting the use of bevacizumab in the treatment of refractory $\mathrm{ABC}$. Therefore, chemotherapy combined with bevacizumab can be considered for patients with primary drug resistance or failure of multi-line therapy without standard drugs. However, prospective and large cohort studies are required to further investigate the efficacy of bevacizumab and determine the patient population that can benefit the most from bevacizumab treatment.

\section{Acknowledgments}

The authors would like to thank the previous researchers for their studies.

Funding: None.

\section{Footnote}

Reporting Checklist: The authors have completed the STROBE reporting checklist. Available at https://dx.doi. org/10.21037/apm-21-2745

Data Sharing Statement: Available at https://dx.doi. org/10.21037/apm-21-2745

Conflicts of Interest: All authors have completed the ICMJE uniform disclosure form (available at https://dx.doi. org/10.21037/apm-21-2745). The authors have no conflicts of interest to declare.

Ethical Statement: The authors are accountable for all aspects of the work in ensuring that questions related to the accuracy or integrity of any part of the work are appropriately investigated and resolved. All procedures performed in this study involving human participants were in accordance with the Declaration of Helsinki (as revised in 2013). The study was approved by the medical ethics committee of Henan Cancer Hospital (No. 2107407) and informed consent was taken from all the patients.

Open Access Statement: This is an Open Access article distributed in accordance with the Creative Commons Attribution-NonCommercial-NoDerivs 4.0 International License (CC BY-NC-ND 4.0), which permits the noncommercial replication and distribution of the article with the strict proviso that no changes or edits are made and the original work is properly cited (including links to both the formal publication through the relevant DOI and the license). See: https://creativecommons.org/licenses/by-nc-nd/4.0/.

\section{References}

1. Harbeck N, Gnant M. Breast cancer. Lancet 2017;389:1134-50. 
2. Folkman J. Angiogenesis. Annu Rev Med 2006;57:1-18.

3. $\mathrm{Hu} \mathrm{X}$, Zhang J, Xu B, et al. Multicenter phase II study of apatinib, a novel VEGFR inhibitor in heavily pretreated patients with metastatic triple-negative breast cancer. Int J Cancer 2014;135:1961-9.

4. Hu X, Cao J, Hu W, et al. Multicenter phase II study of apatinib in non-triple-negative metastatic breast cancer. BMC Cancer 2014;14:820.

5. Lü HM, Zhang MW, Niu LM, et al. Clinical observation of apatinib mesylate for the treatment of multi-drug resistant advanced breast cancer. Zhonghua Yi Xue Za Zhi 2018;98:1246-9.

6. Miller K, Wang M, Gralow J, et al. Paclitaxel plus bevacizumab versus paclitaxel alone for metastatic breast cancer. N Engl J Med 2007;357:2666-76.

7. Pivot X, Schneeweiss A, Verma S, et al. Efficacy and safety of bevacizumab in combination with docetaxel for the firstline treatment of elderly patients with locally recurrent or metastatic breast cancer: results from AVADO. Eur J Cancer 2011;47:2387-95.

8. Eisenhauer EA, Therasse P, Bogaerts J, et al. New response evaluation criteria in solid tumours: revised RECIST guideline (version 1.1). Eur J Cancer 2009;45:228-47.

9. Gonzalez-Angulo AM, Morales-Vasquez F, Hortobagyi GN. Overview of resistance to systemic therapy in patients with breast cancer. Adv Exp Med Biol 2007;608:1-22.

10. Araki K, Miyoshi Y. Mechanism of resistance to endocrine therapy in breast cancer: the important role of PI3K/Akt/ mTOR in estrogen receptor-positive, HER2-negative breast cancer. Breast Cancer 2018;25:392-401.

11. Sikov WM, Dizon DS, Strenger R, et al. Frequent pathologic complete responses in aggressive stages II to III breast cancers with every-4-week carboplatin and weekly paclitaxel with or without trastuzumab: a Brown University Oncology Group Study. J Clin Oncol 2009;27:4693-700.

12. Pernas S, Tolaney SM. HER2-positive breast cancer: new therapeutic frontiers and overcoming resistance. Ther Adv Med Oncol 2019;11:1758835919833519.

13. Bachelot T, Bourgier C, Cropet C, et al. Randomized phase II trial of everolimus in combination with tamoxifen in patients with hormone receptor-positive, human epidermal growth factor receptor 2-negative metastatic breast cancer with prior exposure to aromatase inhibitors: a GINECO study. J Clin Oncol 2012;30:2718-24.

14. Campone M, Bachelot T, Gnant M, et al. Effect of visceral metastases on the efficacy and safety of everolimus in postmenopausal women with advanced breast cancer: subgroup analysis from the BOLERO-2 study. Eur J

Cancer 2013;49:2621-32.

15. Yardley DA, Noguchi S, Pritchard KI, et al. Everolimus plus exemestane in postmenopausal patients with $\mathrm{HR}(+)$ breast cancer: BOLERO-2 final progression-free survival analysis. Adv Ther 2013;30:870-84.

16. André F, O'Regan R, Ozguroglu M, et al. Everolimus for women with trastuzumab-resistant, HER2-positive, advanced breast cancer (BOLERO-3): a randomised, double-blind, placebo-controlled phase 3 trial. Lancet Oncol 2014;15:580-91.

17. Tao JJ, Castel P, Radosevic-Robin N, et al. Antagonism of EGFR and HER3 enhances the response to inhibitors of the PI3K-Akt pathway in triple-negative breast cancer. Sci Signal 2014;7:ra29.

18. Li Q, Wang Y, Jia W, et al. Low-Dose Anti-Angiogenic Therapy Sensitizes Breast Cancer to PD-1 Blockade. Clin Cancer Res 2020;26:1712-24.

19. Folkman J. Tumor angiogenesis: therapeutic implications. N Engl J Med 1971;285:1182-6.

20. Folkman J. Seminars in Medicine of the Beth Israel Hospital, Boston. Clinical applications of research on angiogenesis. N Engl J Med 1995;333:1757-63.

21. Carmeliet P. VEGF as a key mediator of angiogenesis in cancer. Oncology 2005;69 Suppl 3:4-10.

22. Santos LV, Cruz MR, Lopes Gde L, et al. VEGF-A levels in bevacizumab-treated breast cancer patients: a systematic review and meta-analysis. Breast Cancer Res Treat 2015;151:481-9.

23. Delli Carpini J, Karam AK, Montgomery L. Vascular endothelial growth factor and its relationship to the prognosis and treatment of breast, ovarian, and cervical cancer. Angiogenesis 2010;13:43-58.

24. Dear RF, McGeechan K, Jenkins MC, et al. Combination versus sequential single agent chemotherapy for metastatic breast cancer. Cochrane Database Syst Rev 2013;(12):CD008792.

(English Language Editor: L. Huleatt)

Cite this article as: $\mathrm{Lv} \mathrm{H}$, Niu L, Zhang M, Zeng H, Zhao S, Wang J, Sun H, Liu Z, Yan M. Efficacy and safety of bevacizumab-containing therapy for refractory advanced breast cancer: a retrospective study. Ann Palliat Med 2021;10(10):1099711005. doi: 10.21037/apm-21-2745 